\title{
Bouleversement de la conscience historique
}

\section{Dagmar Klose}

Traducteur : Dominique Gélin

\section{OpenEdition}

\section{Journals}

Édition électronique

URL : http://journals.openedition.org/ries/3247

DOI : 10.4000/ries.3247

ISSN : 2261-4265

Éditeur

Centre international d'études pédagogiques

Édition imprimée

Date de publication : 1 mars 1997

Pagination : 67-80

ISSN : 1254-4590

Référence électronique

Dagmar Klose, "Bouleversement de la conscience historique », Revue internationale d'éducation de Sèvres [En ligne], 13 | 1997, mis en ligne le 24 juillet 2013, consulté le 30 avril 2019. URL : http:// journals.openedition.org/ries/3247 ; DOI : 10.4000/ries.3247 


\section{Bouleversement de la conscience historique ? ${ }^{1}$}

Dagmar Klose

\section{Symboles}

Dans Chemnitz, ma ville natale, trône depuis de nombreuses années un monument chargé d'histoire ; c'est lui qui a donné, pendant un certain temps, un autre nom à ma ville natale : Karl Marx. Un crâne, Nischel en dialecte saxon, devait annoncer en place publique, par sa taille considérable, que rien ne pouvait arrêter un socialisme d'airain.

Aujourd'hui je me tourne plutôt, avec tout le respect que je dois à ce que le vieux monsieur de Trèves a apporté à la science, vers des symboles historiques plus subtils. Ils me permettent, avec mes propres erreurs, mes propres ruptures, mais aussi grâce à de nouvelles synthèses et en cherchant les voies qui me paraissent possibles, d'entrer dans un dialogue où il n'y aura ni vainqueur ni perdant. Où il y aura, bien au contraire, une volonté d'écoute réciproque.

Ce qui me touche dans le tableau de Paul Klee, "L'Ange de l'Histoire ", c'est la façon dont l'Ange reste fixé sur le passé, passé dont il ne parvient pas à se détacher bien qu'une tempête l'emporte en permanence, à reculons, vers le futur ${ }^{2}$. Ce qu'il voit semble être plus épouvantable que beau ou même enthousiasmant ; c'est aussi le sentiment de Walter Benjamin qui le voit entouré de la chaîne des événements passés, représentation, pour lui, de la Catastrophe. Mais, contrairement à Walter Benjamin, je ne vois pas uniquement, dans les débris de ces « restes" historiques, un gigantesque tas de ruines; j'aimerais rassembler aussi les débris, pour former un tout. Le temps qui passe guérit les blessures, le fossé se comble, peu à peu.

Benjamin trace, lui aussi, un vaste arc de cercle entre passé et avenir. Le point, fugitif, où ils se rencontrent, représente, sans nul doute, ce que nous appelons le présent. Le nerf vital de notre psychisme prend soin de rétablir, imperturbablement, de nouveaux équilibres, de tracer de nouvelles symétries

1 Cet article a été traduit par Dominique Gélin.

2 Voir l'interprétation de Walter Benjamin proposée par Ralf Konersmann, Erstarrte Unruhe. Walter Benjamins Begriff der Geschichte (L'inquiétude figée. La conception de l'histoire de Walter Benjamin), Frankfurt/Main, Ed. Fischer, 1991, p.121. 
entre le passé et l'avenir, sur l'axe du temps de la vie, l'axe du temps biographique $^{3}$. Et le travail de la mémoire est là pour répondre, avec complaisance, à ce besoin qui est en nous, d'atténuer le plus possible toute tension entre monde intérieur et monde extérieur ${ }^{4}$.

Comme l'histoire, qui était, à l'instant encore, notre présent et au beau milieu de laquelle on pouvait nous voir, est impénétrable! Bien que nous ayons, en général, une perception sélective des choses, il semble très difficile de faire la part, placé devant l'ensemble des événements, de ce qui est éphémère, passager, et de ce qui sera plus durable, qui contient en germe quelque chose de nouveau. A ce niveau, au niveau "des événements de courte durée ", ainsi que les nomme le maître français Braudel, on pense que le plus opportun est de s'en tenir aux faits qui permettent d'avoir une certitude suffisante.

\section{Tout n'a-t-il pas déjà été dit ?}

Six ans aprés l'intégration de la RDA dans la République fédérale d'Allemagne, le problème posé par le personnel dirigeant a été, pour l'essentiel, réglé ; des structures stables ont été mises en place. Après une courte phase de divergence, où l'historiographie de la RDA avait tenté d'affirmer son originalité, les personnalités les plus en vue de cette historiographie ont été «abgewickelt » ${ }^{5}$, à quelques exceptions près. Un petit nombre d'historiographes, plus jeunes et moins marqués, doivent apprendre, en temps limité, donc dans des conditions de travail difficiles, à se mettre au niveau scientifique exigé à l'Ouest, et, ce qui est à peine moins difficile, à maîtriser l'art du management et du marketing scientifique. Conquérir le marché scientifique pose problème, non seulement du fait du manque d'expérience, mais aussi parce que le réseau de l'historiographie de l'Ouest s'est, entretemps, élargi vers l'Est ${ }^{6}$.

3 Le concept de symétrie est dû à l'anthropologue Wessel. Pour plus ample information : Karl-Friedrich Wessel, "Über die den Individuen möglichen Vermittlungen zwischen Vergangenheit und Gegenwart - Oder : Gibt es eine individualisierte Geschichte? " (Au sujet des rapports possibles que l'individu peut établir entre le passé et le présent - Ou bien : Existe-t-il une histoire individualisée ?), in : Dagmar Klose, Vergangenheit - Geschichte - Psyche. Ein interdisciplinäres Gespräch (Passé - Histoire - Psychisme. Dialogue interdisciplinaire), Idstein Ed., Uwe Uffelmann, 1993, p. 23-33.

4 Voir pour un exposé détaillé : Luc Ciompi, Die Entstehung von Zeit, Raum und psychischen Strukturen, (Monde extérieur - monde intérieur. Genèse du temps, de l'espace et des structures psychiques), Göttingen, 1988.

5 Ce terme a été utilisé couramment à partir de 1991 pour désigner le processus, systématiquement mis en place pour raison d'" épuration " idéologique, de mise à l'écart des responsables ou des enseignants en poste dans les universités ou établissements de l'ancienne RDA (N.D.L.R.).

6 L'historiographie allemande depuis la réunification est bien documentée. Consulter, parmi d'autres :

- Rainer Eckert, Wolfgang Küttler, Gustav Seeber, Krise - Umbruch - Neubeginn. Eine kritische und selbstkritische Dokumentation der DDR-Geschichtswissenschaft (Histoire de la RDA: crise - renversement - renouveau. Documentation critique et autocritique), Stuttgart, 1992. 
La réforme de l'enseignement de l'histoire à l'université a plutôt suivi la distinction classique entre différentes époques que l'idée de créer des chaires «non-conventionnelles" comme par exemple l'histoire des cultures et des mentalités.

Mais pourquoi devait-on alors réformer spécialement la formation des enseignants ? Là aussi, les structures ouest-allemandes ont servi de modèle dans tous les Länder de l'Est. Elles ont étouffé toutes les tentatives qui avaient pour objectif de continuer à soutenir, à l'Ouest et à l'Est, les idées novatrices pour, finalement, revenir aux schémas conventionnels, qui sont pourtant déjà critiqués dans les anciens Länder. Le système de formation en deux phases, correspondant à ces structures, a été repris alors qu'il était l'objet de vives controverses, sans tenir compte des expériences alternatives à l'échelon européen ${ }^{7}$.

- Christoph Kleßmann, Martin Sabrow, "Aus Politik und Zeitgeschichte " (Extraits de la politique et de l'histoire contemporaine), Supplément à l'hebdomadaire Das Parlament, Suppl. 39/96 du 20.9.1996, p. 3-14.

- Ulrich Neuhäußer-Wespy, "Geschichtswissenschaften unter der SED-Diktatur. Die Durchsetzung der Parteilinie in den fünfziger Jahren " (Les sciences historiques sous la dictature du Parti socialiste unifié d'Allemagne. Comment la ligne du Parti s'est imposée dans les années cinquante), Ibid. p. 15-21.

- Ilka-Sascha Kowalczuk, "Die DDR-Historiker und die deutsche Nation » (Les historiens de la RDA et la nation allemande), Ibid. p.22-30 ; Paradigmen deutscher Geschichtswissenschaft. Ringvorlesung an der Humboldt-Universität zu Berlin (Paradigmes de l'historiographie allemande. Cycles de conférences de l'université Humboldt), Berlin, 1994.

- Konrad H. Jarausch, éd.,Zwischen Parteilichkeit und Professionalität. Bilanz der Geschichtswissenschaft der DDR (Entre professionnalisme et esprit partisan. Bilan des sciences historiques de la RDA), Berlin, 1991.

- Konrad H. Jarausch, Matthias Midell, éd., Nach dem Erdbeben. (Re-)Konstruction ostdeutscher Geschichte und Geschichtswissenschaften (Après la secousse tellurique. Re-construction de l'histoire et de la recherche historique est-allemandes), Leipzig, 1994.

- Heiner Timmermann, éd., "DDR-Forschung. Bilanz und Perspektiven " (La recherche sur la RDA. Bilan et perspectives), in: Dokumente und Schriften der Europäischen Akademie Otzenhausen (Textes et documents de l'Académie européenne de Otzenhausen), Berlin, 1995, vol. 76.

- Udo Wengst, "Deutsche Geschichte nach 1945 / Teil I und II" (Comptes rendus littéraires: I'histoire de l'Allemagne après $1945 / 1^{\text {re }}$ et $2^{\mathrm{e}}$ parties), Geschichte in Wissenschaft und Unterricht (Recherches historiques et enseignement de l'histoire), GWU, 47 (1996) 9,10, p. 539-558, 620-639.

7 Le "modèle de Postdam concernant la formation des enseignants ", conçu pour l'université de Postdam, constitue une exception; il accorde une grande importance à l'orientation professionnelle tout en conservant le système de formation en deux phases. Cependant les idées novatrices menacent de se dessécher, parce qu'elles n'inspirent guère la majorité de ceux qu'elles devraient combler. La thèse opposée, selon laquelle ces conceptions sont utopiques, risque alors de s'imposer.

Voir sur ce sujet, Wolfgang Edelstein, Ulrich Herrmann, "Universität Postdam. Modell der Lehrerbildung " (L'université de Postdam. Le modèle de la formation des enseignants), Strukturkommission Lehrerbildung des Gründungssenats der Universität Postdam (commission structurelle du Sénat fondateur de l'université de Postdam ayant pour objet la formation des enseignants), Université de Postdam, brochure, sept. 1992. 
La réforme complète de l'enseignement de l'histoire dans les nouveaux Länder a été accomplie en respectant les axes importants des plans cadres des Länder partenaires de l'Ouest, bien que, la plupart du temps, des commissions réellement paritaires aient été réunies. Mais les pédagogues est-allemands manquaient à peu près complètement d'expérience pour aborder les questions posées par la didactique. En RDA, de telles prémisses étaient données, d'emblée, par la doctrine politique à destination des écoles. Ce manque de compétences didactiques dans la discipline fait qu'il est très difficile de reconnaître les prémisses didactiques sur lesquelles se fondaient les différents ouvrages pédagogiques et modèles de cours, et d'en peser le pour et le contre. Ce déficit se manifeste aussi par le fait que les enseignants manquent de confiance en eux-mêmes, qu'ils ne sont pas capables de donner les raisons qui les ont guidés dans leurs choix didactiques, si l'on considère les nouveaux espaces de liberté offerts pour bâtir un curriculum individuel, alors que ces espaces de liberté étaient justement ce à quoi ils aspiraient dans le passé.

Le manuel d'histoire uniformément utilisé dans toute la RDA a été remplacé, sans aucune exception, par tout un éventail de manuels ouestallemands, de façon assez analogue à ce qui s'était passé en 1945, devant la grande misère des ouvrages scolaires. Ils ont, dans un premier temps, été très favorablement accueillis, non seulement à cause de leur présentation attrayante mais aussi parce qu'ils permettaient de se libérer du carcan idéologique, parce qu'ils prenaient en compte les aspects quotidiens de l'histoire et qu'ils proposaient des tâches sous forme d'activités. Certes la recherche de liens entre les événements a correspondu dans un premier temps à la façon de penser habituelle, dans le cadre des structures officielles; mais c'est là aussi une nécessité de la théorie de l'histoire et de la psychologie de l'apprentissage. On a cependant le sentiment qu'on ne lui a toujours pas reconnu l'importance qu'elle devrait avoir quand il s'agit de passer à la pratique de l'enseignement.

La décision de savoir jusqu'à quel point des enseignants historiens, impliqués dans le système en place jusque-là, devaient quitter leurs fonctions ou bien pouvaient devenir, après recyclage, des enseignants "acceptables » dans cette nouvelle situation, a été prise de différentes façons selon les Länder. On a, en règle générale, défini différentes catégories, d'après lesquelles on a pu ensuite évaluer la qualification des enseignants dans le domaine politique et dans la discipline. Mais il y eu des différences selon les Länder, les enseignants devant, dans certains cas, faire acte de candidature pour les postes nouvellement mis à disposition, après avoir reçu leur congé et avoir été inscrits sur une "liste d'attente » alors que, dans d'autres cas, l'évaluation avait lieu sans qu'ils cessent d'accomplir leur tâche.

Entre-temps, la situation de l'enseignement de l'histoire s'est, au moins si on la considère de l'extérieur, largement alignée sur celle de l'Ouest, 
ce qui signifie aussi qu'on en a repris non seulement les aspects positifs, mais aussi les carences ${ }^{8}$.

\section{Processus structurels et évolution des mentalités}

En cette période intermédiaire les contours deviennent flous, faisant apparaître davantage le côté éphémère des interprétations actuelles.

Cette situation, où l'on compare culture historique et conscience historique à l'intérieur d'une même nation, en explorant plus profondément les mécanismes qui président à la formation de la conscience historique, est unique dans l'histoire. Depuis 1989, un nombre non négligeable de recherches ont été faites concernant les dispositions d'esprit des Allemands, le plus souvent sous la responsabilité de scientifiques ouest-allemands, avec la collaboration de collègues est-allemands, les personnes interrogées étant de préférence est-allemandes. Ils devaient, avant tout, fournir des renseignements sur les processus de socialisation des Allemands de l'Est dans le cadre du nouveau système, et sur la manière dont ils y prenaient place, en tant qu'individus. Les propos recueillis font apparaître, dans leur totalité, des éléments contradictoires, contraires même ${ }^{9}$. Si on va vraiment au fond des choses, ce sont les thèses suivantes qui se dégagent: le caractère traditionnellement autoritaire de l'État, hérité de l'époque impériale, a continué à jouer un rôle important dans la société de la RDA et "on y a conservé plus de vestiges de l'ancienne société que dans la République fédérale, qui, elle, s'est occidentalisée plus rapidement ${ }^{10}$ ». Mais on reconnaît en revanche aux Allemands de l'Est une mentalité autonome en relevant les transformations importantes qu'a connues la vie sociale en RDA. Ce serait là l'une des raisons pour lesquelles, pense-t-on, le processus d'unification des mentalités se déroulerait d'une manière plus compliquée que prévu ${ }^{11}$.

Il y a des différences considérables d'un travail de recherche à l'autre, aussi bien en ce qui concerne la théorie que la méthode employée, et, par voie

8 Bodo von Borries, Das Geschichtsbewußtsein Jugendlicher. Eine repräsentative Untersuchung über Vergangenheitsdeutungen, Gegenwartswahrnehmungen und Zukunftserwartungen von Schülerinnen und Schülern in Ost- und Westdeutschland (La conscience de l'histoire chez les jeunes. Recherche sur les représentations des élèves dans l'Allemagne de l'Est et de l'Ouest : comment ils interprètent le passé, leur perception du présent, ce qu'ils espèrent de l'avenir), Weinheim et Münich, 1995.

9 Werner Weidenfeld, éd., Deutschland. Eine Nation - doppelte Geschichte. Materialien zum deutschen Selbstverständnis (L'Allemagne. Une nation - une double histoire. Documentation : pour aider les Allemands à mieux se comprendre eux-mêmes), Cologne, 1993.

10 Hartmut Kaelble, Jürgen Kocka, Hartmut Zwahr, éd., Sozialgeschichte der DDR, (Histoire sociale de la RDA), Stuttgart, 1994, p. 550 et 551.

11 Thomas Metzinger, éd., Bewssusstsein. Beiträge aus der Gegen wartsphilosophie (Conscience. Les apports de la philosophie de Présent), Paderborn, 2e éd., 1996. 
de conséquence, entre les interprétations proposées, qu'elles soient globales ou différenciées. On serait enclin à faire la remarque que bien des enquêtes nous renseignent, avant tout, sur le niveau de conscience de leurs auteurs, niveau de conscience qu'ils laissent transparaître derrrière leur interprétation avec un réalisme naïf dont on ne peut guère être dupe. C'est tout à fait compréhensible du point de vue de la philosophie et de la psychologie de la conscience ; toutes nos réflexions se situent bien entendu dans la perspective de notre propre espace de conscience. Dans ce domaine sensible de la recherche, il est indispensable de débattre des subjectivités et pas uniquement lorsqu'il s'agit de démarches explicitement herméneutiques. En outre, il faut bien voir que les enquêtes faites selon les méthodes quantitatives font généralement apparaître plutôt des symptômes superficiels que des structures profondes.

Les enquêtes comparant la conscience historique chez les enfants et les adolescents à l'Est et à l'Ouest sont instructives. On s'attendait à des différences considérables, l'histoire étant précisement la discipline où les divergences sont cultivées, et pourtant B. von Borries, l'un des rares didacticiens de l'histoire en République fédérale à travailler de façon empirique, a constaté qu'on trouvait dans ce domaine plus de choses rapprochant que séparant enfants et adolescents. Cela pourrait tenir à un relatif manque d'efficacité de la transmission du savoir dans cet enseignement, malgré les apports de la didactique. Les expériences vécues dans le présent n'y trouvent pratiquement aucun contrepoids. La conscience de l'histoire est manifestement beaucoup plus marquée par la réalité sociale, l'actualité dans le monde, l'expérience vécue, la biographie propre. Ce sont elles qui déterminent la recherche actuelle du sens, quête qui n'est guère satisfaite par la façon dont elle est enseignée. On peut probablement relier à cela une autre explication : le fait que la tradition culturelle commune ait conservé une influence plus profonde et que le sens attribué à l'histoire pendant quarante-cinq ans par les institutions de la RDA n'a pu que partiellement extirper. D'un autre côté, la façon ambitieuse dont elle est présentée à l'Ouest, dans la "multiplicité des perspectives ", fait qu'on assiste rapidement, par suite du manque de compétence en histoire à l'âge scolaire, à une métamorphose des explications et des interprétations pour aboutir à quelque chose d'arbitraire ; ou bien on s'en tient même, dans certains cas, à une manière positiviste de voir les choses.

Pendant que, d'un côté, les différences semblent rapidement s'estomper entre Est et Ouest en ce qui concerne la conscience historique des jeunes, du fait que leur expérience vécue en RDA a été de plus courte durée et que leur perspective de vie les emmène bien au delà, il n'en va pas de même en ce qui concerne l'évolution des mentalités chez les pédagogues dont la tâche est la socialisation par l'enseignement de l'histoire. On est amené à croire que cette catégorie professionnelle, structurellement proche du système, a fréquemment intériorisé, au moins partiellement, au cours d'une formation profondément marquée idéologique- 
ment, les contours du tableau que les autorités brossaient de l'histoire, même si cela se retrouvait peu dans les transparents utilisés en cours.

B. von Borries a étudié, en 1993, les conceptions didactiques des enseignants est-allemands. Quelle orientation donner au savoir et à la discipline enseignée, à la problématique et aussi à la participation émotive aux événements : ces composantes importantes d'une "attitude amicale envers les élèves ${ }^{12}$ » sont la manifestation superficielle de symptômes dont les structures profondes ont été analysées par R. Schörken dans son travail sur les principaux mécanismes didactiques du manuel d'histoire en RDA, pour tous les niveaux ${ }^{13}$.

Il est indéniable que l'image que la RDA présentait de l'histoire était, au plus haut point, celle d'une histoire porteuse de sens. La dimension sociale de la lutte des classes lui conférait un sens universel, en identifiant l'ensemble du processus historique à la juste lutte des opprimés contre leurs oppresseurs. On n'héroïsait plus de "grands hommes", appartenant en règle générale à la classe des dirigeants, comme dans les autres périodes de l'histoire allemande; les "héros" sortaient, au contraire, des masses. Cet objectif d'identification intervenait sur un terrain très sensible : essentiellement les jeunes enfants, très réceptifs sur le plan émotionnel et entrant très facilement, comme chacun sait, dans le schéma dichotomique du Bien et du Mal. L'enseignement de l'histoire, en s'appuyant sur la sensibilité des enfants, leur sentiment de justice et le besoin de trouver un sens à l'histoire, essayait de contribuer à l'identification aux idéaux socialistes et pouvait tout à fait leur donner le sentiment de se situer dans la tradition des idées progressistes. La recherche du sens de l'histoire et de la justice semblent être des besoins très résistants, qui conditionnent la manière dont l'histoire est reçue à l'Est de l'Allemagne.

L'alliance d'une « logique de l'histoire » et du recours à l'émotionnel ${ }^{14}$ correspondait d'autant plus aux modes d'appropriation des enfants et des jeunes que, d'une part, les prémisses théoriques données et le modèle explicatif d'une structure relativement simple, dénommé marxiste-léniniste, n’étaient pas difficiles à assimiler pour filles et garçons et que, d'autre part, la force de la participation émotive, dans son mode de transmission, représente en général un élément significatif dans la construction de la conscience historique.

Cette façon vivante de transmettre les connaissances, libérée de toute idéologie et liée à une mise en œuvre des dernières théories de l'apprentissage, est tout à fait digne d'être conservée.

12 Bodo von Borries, note 8, op. cit. p. 324 et suiv.

13 Rolf Schörken, « Didaktische Mechanismen in der DDR- Lehrbuchwerk Geschichte 5-10 " (Mécanismes didactiques en RDA. Les manuels d'histoire 5-10), GWU 2/1992, p. 94 et suiv.

14 Ibid. p. 99 et suiv. 
Ce bouleversement de l'enseignement de l'histoire a été avant tout perçu, de l'extérieur, comme donnant le signal d'un nouveau départ. Mais la situation dans laquelle se trouvaient et se trouvent les enfants et les jeunes, du point de vue des mentalités, est beaucoup plus compliquée parce qu'il n'est justement pas possible de séparer son étude du monde de la vie.

Ces bouleversements dans l'enseignement de l'histoire se sont traduits pour les élèves par une transformation brutale du cours d'histoire. Les vérités traditionnelles perdirent subitement toute valeur. On ne voyait aucun nouveau système permettant de s'orienter, ou bien ils n'étaient pas acceptables. Les nouvelles propositions offertes éveillent la méfiance, méfiance entretenue par les expériences vécues. Le fait que les structures du « réalisme socialiste aux couleurs de la RDA " soient devenues poreuses, qu'elles se soient effondrées comme un château de cartes, a signifié pour tous ceux qui étaient concernés par l'enseignement de l'histoire, quelle que soit leur implication, plus ou moins grande, dans la société socialiste, un changement abrupt de paradigmes, très lourd à supporter psychiquement. Cette tension était tout autant liée au brusque changement des contenus de l'enseignement et, ce qui est plus grave, des interprétations qu'on en donnait, qu'au doute, doute de soi-même, doute quant à la crédibilité des différents enseignants de la discipline, doute également quant au rôle que l'on jouait soi-même concernant la place de l'école dans la société. Comment les enseignants ont-ils été acceptés par leurs élèves après le tournant historique ? Cela dépendait, au plus haut degré, de la manière dont ils réussissaient à intégrer les questions de leurs élèves dans leur propre réflexion, leurs propres démarches, et à les faire participer à leurs propres recherches. Pour les uns, continuer à être accepté, au delà du renversement de situation, par les filles et les garçons, conserver leur confiance, a été plus facile que pour d'autres, ceux qui ont changé leur fusil d'épaule ou ont réécrit leur propre histoire qui devenait, du jour au lendemain, celle d'une victime. D’une façon générale, enfants et adolescents se sont révélés être des partenaires fiables, bien plus honnêtes, il s'en faut de beaucoup, que les démêlés entre collègues auxquels on a pu assister dans plus d'un établissement.

Pour beaucoup d'adolescents, cela a pesé non seulement sur leurs rapports avec l'école mais aussi, lourdement, sur leurs rapports avec leur famille. Par exemple, lorsque les parents, qui s'étaient accommodés du régime de la RDA ou même qui s'y étaient fait une place, commencèrent à avoir peur de perdre leur emploi et leur statut dans la société, ou lorsque cela devint réalité.

Cette phase du bouleversement historique, dans le domaine scolaire et le domaine privé, a échappé jusqu'ici, à quelques exceptions près, aux recherches scientifiques. C'est regrettable mais, également, compréhensible: d'une part, parce que tout le secteur scientifique de l'Est de l'Allemagne touchant à la sociologie et aux sciences humaines s'était, presque sans exception, 
discrédité ; d'autre part, parce que, du point de vue de l'Ouest, on a bien souvent essayé de faire disparaître les pratiques sociales sous des constructions théoriques qui ne permettaient ni de les recenser de façon adéquate ni d'en donner une explication satisfaisante.

\section{Une occasion manquée ?}

Cette question, qui restera soumise à l'indulgence des générations suivantes, se pose sur l'axe du temps de la «longue durée». Pourtant, la question de savoir ce qui restera et ce qu'il adviendra ne nous laisse pas de répit, à nous qui vivons aujourd'hui.

L'analyse sociologique du processus de la réunification oscille entre le concept de «colonialisation» et celui de «démocratisation», ou bien elle revient au concept, exempt de tout jugement de valeur, de « transformation ${ }^{15}$ ».

La thèse générale suivante peut se rapporter aussi à la situation des instances spécialisées dans les domaines de la recherche et de la transmission des connaissances en histoire et, en particulier, ceux de la formation des enseignants et de l'enseignement de cette discipline.

"Étant donné qu'on a transféré des solutions qui remontent, pour la plupart, à la résolution de problèmes et à la recherche de compromis entre différents intérêts, tels qu'ils se posaient à la fin du XIX et au début du $\mathrm{Xx}^{\mathrm{e}}$ siècle, il y a peu d'espoir qu'ils aident l'Allemagne de l'Est à se développer et combler son retard. Le projet, conservateur de par sa forme, poussa plutôt à sous-estimer les besoins de réforme... ${ }^{16}$ "

Se demander maintenant quelles auraient pu être les alternatives, paraît être une méthode ahistorique. Car seule une très petite partie de la population de la RDA voulait la confédération. La majorité voulait le rattachement, rapide, à la République fédérale. Ce qui rend d'autant plus séduisante la thèse que les spécialistes dans le domaine des sciences humaines, et c'était la majorité, n'auraient jamais été capables de se réformer eux-mêmes dans un laps de temps court.

Se réformer, de l'intérieur, exige qu'on réfléchisse non seulement à sa propre biographie mais aussi à la structure épistémologique et à la genèse de la discipline dont on est spécialiste, dans différents systèmes socioculturels. On n'a seulement commencé à faire sérieusement appel à ce modèle qu'à la suite de

15 Fritz Volmar, Wolfgang Dümcke, "Kritische Zwischenbilanz der Vereinigungspolitik. Eine unerledigte Aufgabe der Politikwissenschaft " (Bilan critique intermédiaire de la politique de réunification. Les sciences politiques n'ont pas achevé leur tâche), Aus Politik und Zeitgeschichte (Extraits de la politique et de l'histoire contemporaine), vol. 40/96, p. 43.

16 Helmut Wiesenthal, «Die neuen BundesLänder als Sonderfall der Transformation » (Les nouveaux Länder, cas particuliers de la Transformation), Ibid. p. 54. 
l'effet de choc et du contraste avec les autres modèles occidentaux. Il permet de filtrer certains stades: choc, ou même, dans certains cas, bouleversement; refoulement et destruction, en même temps qu'on adopte de nouveaux savoirs, apportés par d'autres systèmes ; concurrence des systèmes épistémologiques et des systèmes de savoirs, et stagnation apparente ; intégration et différenciation ; distance critique par rapport aux deux systèmes scientifiques; innovation. On pourrait faire également le tracé d'un autre enchainement de phases, analogue : celui décrit par le balancier du sentiment que l'on a de sa propre valeur ${ }^{17}$.

C'est ainsi que les processus de réforme vraiment intéressants auront lieu, si tant est qu'ils aient jamais lieu, avec un décalage dans le temps et, avant tout, là où les gens de l'Est et de l'Ouest travaillent en présence les uns des autres et communiquent entre eux. La présence de spécialistes étrangers a également, en règle générale, une influence bénéfique. Cela tient, bien entendu, au fait qu'ils voient les choses de l'extérieur; ce qui leur permet de se mettre hors de portée des perturbations qu'apporte une mauvaise ambiance de travail, sous l'influence de malentendus, de préjugés et, parfois, de provocations interallemandes délibérées.

De façon exemplaire et avec beaucoup de tact vis-à-vis des rites qui accompagnent, dans la corporation des historiens, l'art de mettre ce qui est négatif sur le compte d'autrui, de le rendre responsable des erreurs commises, le comité directeur de la conférence sur la didactique de l'histoire a très tôt pris l'initiative d'organiser des rencontres germano-allemandes, que ce soit au niveau de la discipline ou à titre privé, et abordé les thèmes importants de la théorie de la didactique de l'histoire lors de colloques, dans des projets, et des publications. Et il ne fait aucun doute que les préjugés, des deux côtés, n'ont pas réussi à compromettre la chance, unique dans l'histoire, de pouvoir tirer un double profit théorique de l'analyse de la conscience historique dans une période de bouleversement historique ${ }^{18}$. Cela a permis de faire avancer la recherche scientifique sur la problématique identitaire, sur l'aspect socioculturel et les caractères spécifiques aux générations, avant tout ${ }^{19}$. La réflexion sur les critères identitaires, acceptables pour

17 Mitchell G. Ash décrit cette opportunité de retour sur soi dans son exposé " Wissenschaftswandel in historischen Umbruchsituationen" (Évolution des sciences dans les situations de bouleversement historique) (1933, 1945, 1989), in : Ideologie und wissenschaftliche Verantwortung. Zielsetzungen und Praxis von Forschung, Lehre und Studium der Geschichtswissenschaft an der PH "Karl Liebknecht " Postdam und am Institut der Universität Postdam (Idéologie et responsabilité scientifique. Objectifs et pratique de la recherche, de l'enseignement et des études historiques à l'institut de formation pédagogique "Karl Liebknecht " à Postdam et à l'institut d'histoire de l'université de Postdam), Luise Schorn-Schütte, éd., Actes de la session du 5.7.1995, Postdam, 1996, p. 31. 18 Ce travail constructif se fait sous la direction du premier président, Uwe Uffelmann (Heidelberg) et du deuxième président, Berndt Mütter (Oldenburg).

19 Jochen Huhn, "Historische Identität als Dimension des Geschichsbewußtseins " (L'identité historique comme dimension de la conscience historique) in: Identitätsbildung und Geschichtsbewußtsein nach der Vereinigung 
tous les Allemands, a aussi enrichi la théorie de la didactique de cette discipline ${ }^{20}$. Le rapport entre la région, la nation et le monde a fait l'objet d'une thématisation didactique; le petit espace, qui prend une plus grande importance du fait de la constitution de nouveaux Länder, a refait son apparition sur la table d'étude du département d'histoire. Cependant, et ce n'est pas là l'aspect le moins important, on a toujours cherché à savoir ce qui valait la peine d'être conservé dans la méthodologie historique de la RDA. Mais les temps ne sont pas encore mûrs pour des préoccupations de ce genre ${ }^{21}$.

L'objet essentiel de la didactique de l'histoire, c'est-à-dire la façon dont se construit la conscience de l'histoire, dans les conditions imposées par chacune des deux cultures historiques, constitue, sur ces thèmes du bouleversement et de la réunification de deux systèmes complètement différents, un véritable Eldorado pour la recherche scientifique.

C'est précisément derrière de soi-disant paradoxes, superficiels, que se cachent les nouvelles découvertes et les points de départ de processus innovants. Le «syndrome d'insatisfaction » qui accompagne, du point de vue de l'Ouest,

Deutschlands (Après la réunification: comment se constitue l'identité et se forme la conscience historique des Allemands), Weinheim, 1993, p. 9-34.

- Également, Berndt Mütter, «Identitätsbildung - Identitätsrevision in Deutschland. Das Beispiel des geisteswissenschaftlichen Konzepts der Erwachsenenbildung " (Une identité qui se constitue, une identité qu'on révise en Allemagne : l'exemple du concept, venu des sciences humaines, de la formation des adultes), Erich Weniger, Ibid. p. 35-57.

- Dagmar Klose, "Prägungen und Wandlungen ostdeutscher Identitäten " (Caractères et évolutions des identités en Allemagne de l'Est), Extraits de la politique et de l'histoire contemporaine, op. cit., suppl. 41/94, p. 3-11. - Uwe Uffelmann, « Identitätsbildung und Geschichtsdidaktik » (Caractères et évolutions des identités en Allemagne de l'Est), Ibid. p. 12-20.

- Horst Kuss, « Historisches Lernen im Wandel. Geschichtsdidaktik und Geschichtsunterricht in der alten und neuen Bundesrepublik » (L'apprentissage de l'histoire change : didactique et enseignement de l'histoire dans l'anciennne et la nouvelle République fédérale), Ibid. p. 21-30.

- Wendelin Szalai, « Die DDR-Geschichtsmethodik im Spannungsfeld zwischen äußerer und innerer Disciplinierung ») (Comment la méthodologie de l'histoire de la RDA se discipline de l'intérieur et de l'extérieur : tension entre ces deux pôles), Ibid. p. 31-39.

20 Uwe Uffelmann, "Spielräume für Identitätsbildung im historisch-politischen Lernen ? » (Quelle marge reste-t-il pour la constitution de l'identité dans les apprentissages historiques et politiques ?) in : Après la réunification: comment se constitue l'identité et se forme la conscience historique des Allemands, Uwe Uffelmann, éd., loc. cit. p. 228-256.

21 Berndt Mütter, "Geschichte der deutschen Geschichtsdidaktik in der Epoche der Teilung 1945- 1990. Perspektiven einer gesamtdeutschen Geschichtsdidaktik " (Histoire de la didactique de l'histoire à l'époque de la division de l'Allemagne, de 1945 à 1990. Quelles perspectives pour une didactique de l'histoire dans le pays réunifié ?) in: Historisches Lernen im vereinten Deutschland. Nation - Europa - Welt (Apprendre l'histoire dans l'Allemagne réunifiée : la nation, l'Europe, le monde), Weinheim, p. 96-123, 1994.

- Berndt Mütter, Uwe Uffelmann, éd., Regionale Identität im vereinten Deutschland. Chance und Gefahr IIdentité régionale dans l'Allemagne réunifiée. Chances et dangers), Weinheim, 1996. 
cette grande transformation, constitue l'un de ces paradoxes, que l'on explique généralement, chez les Allemands de l'Est, par la perte du sentiment de leur propre valeur et le sentiment que les chances d'avoir un travail et une vie satisfaisants sont réduites ${ }^{22}$.

C'est sans doute valable pour les premières années de ce tournant historique. A l'heure actuelle, le balancier passe plutôt de l'autre côté. Le syndrome d'insatisfaction s'exprime à présent, de manière constructive, dans une conscience nouvelle de soi, de sa propre valeur, qui amène les Allemands de l'Est à défendre avec véhémence l'histoire de leur vie propre, l'histoire telle qu'ils l'ont vécue, en l'opposant à l'histoire du système politique. On ne peut pas enseigner aujourd'hui l'histoire de l'Allemagne dans les nouveaux Länder sans tenir compte de cette sensibilité ${ }^{23}$.

La manière dont l'histoire de l'Allemagne est enseignée dans les établissements scolaires permet de donner un exemple concret de cette évolution ${ }^{24}$. Lorsqu'on exigea que le document « La question allemande dans l'enseignement de l'histoire ", datant de 1978, soit examiné par la conférence des ministres de l'Éducation, à l'occasion du cinquième anniversaire de la réunification en pensant qu'un consensus pourrait s'établir sur cet amendement, on ne vit pas seulement resurgir dans le groupe d'experts les grands débats de l'ancienne République fédérale - la question de la responsabilité de la guerre de 1914, la terminologie concernant 1933 : "prise de pouvoir par la force » ou «transmission du pouvoir ", le débat sur les concepts de "nation » et d' "identité nationale ». Cette fois on vit aussi les Allemands de l'Est, qui donnaient au début l'impression d'avoir "en grande partie perdu l'usage de la parole », se défendre avec de plus en plus de véhémence, soulignant que le projet présentait quarante ans d'un passé où deux États allemands avaient été séparés et avaient existé séparément, dans une perspective très empreinte de la supériorité occidentale. Le débat autour de la question allemande dans l'enseignement de l'histoire trouva une fin provisoire à l'automne 1996, à Wittenberg. On n'avait que trop clairement l'intention de mettre en place une certaine image de l'histoire, une certaine façon d'en concevoir la pédagogie, autant de processus qui rappelaient, par une sorte de fatalité, aux participants est-allemands précisément la façon de procéder de l'ancien temps. On voyait bien qu'il manquait, fort malheureusement, la perspective est-allemande. A nouveau une peinture en noir et blanc des choses, avec une dominance ouest-allemande? On obtint au moins un résultat : on avait pris la dimension du problème et on pouvait espérer avoir pris une sage

22 Helmut Wiesenthal, note 16, p. 52.

23 Rüdiger Thomas, "Aufklärung statt Abrechnung : Anmerkung zum Umgang mit der DDR-Geschichte " (Former et informer au lieu de régler des comptes : note sur les rapports qu'on peut entretenir avec l'histoire de la RDA), Werner Weidenfeld, éd., Voir note 9, op. cit. p. 266.

24 Sabine Etzold, "Ein deutsches Requiem » (Un requiem allemand), Die Zeit, $n^{\circ} 44$ du 25.10.1996, p.45. 
décision en faisant en sorte que la question de l'enseignement de l'histoire allemande ne soit plus de la compétence de la bureaucratie scolaire, qu'elle soit confiée, au contraire, aux historiens.

Deux exigences ont continué à s'imposer dans le domaine de l'historiographie : il était indispensable, d'une part, de savoir reconnaître les sensibilités ; l'histoire des deux Allemagnes devait, d'autre part, être nécessairement saisie dans la perspective d'une interdépendance différenciée, en tenant compte de l'environnement historique, dans le cadre des événements européens et internationaux. "Cette discussion, qui dure toujours, ne suit pas le tracé de l'ancienne frontière entre l'Est et l'Ouest. Elle ne se déroule pas non plus exclusivement dans un cadre scientifique... Les débats, qui se poursuivent à l'heure actuelle, autour du standard scientifique des travaux des historiens depuis 1989 , ont en général la caractéristique suivante : ils servent souvent autant à fournir une identité à chacun des différents groupes d'historiens, à représenter leurs intérêts, qu'à une fructueuse discussion sur la pratique du travail d'historien sous la dictature ${ }^{25}$ ".

En cherchant à sonder plus profondément les mécanismes de la conscience historique individuelle, nous nous heurtons constamment aux questions, fondamentales, de notre compréhension de l'histoire et aux problèmes se rapportant à l'histoire de l'histoire, en tant que discipline scientifique. Le comportement des Allemands de l'Est qui, le cas n'est pas rare, est ressenti du côté occidental comme paradoxal, prend justement racine là où certaines conceptions de l'histoire, le point de départ de certains travaux de recherche trouvent leur limite. "A l'avenir, le passé appartiendra-t-il à une science sociale revisitée ou à une histoire culturelle conquérante? » demande Hans-Ulrich Wehler, qui poursuit: "Sur le plan méthodologique nous nous rendions bien compte, abstraitement, que l'être humain n'agit en aucune façon uniquement par intérêt, mais, que ce sont, toujours, "l'image" qu'il a du monde et les traditions culturelles qui lui montrent le chemin; la perception de ce qu'il est convenu d'appeler la réalité et la façon de l'interpréter; les mentalités et les habitus; tout ce qui va être son environnement social par excellence. En d'autres termes, ce ne sont plus les structures socio-économiques, les intérêts, qui sont considérés comme les éléments moteurs privilégiés, mais plutôt les valeurs et les changements dans les mentalités. C'est à cela qu'on a recours, avant tout, là où les dures contraintes des sociétés modernes laissent subsister des espaces de liberté pour l'action individuelle responsable ${ }^{26}$. »

Cette démarche encouragera tous ceux qui ont eu recours jusque-là aux méthodes empiriques ou même qui, grâce à un travail de réflexion théorique et

25 Christoph Kleßmann, Martin Sabrow, "Zeitgeschichte in Deutschland nach 1989 " (L'histoire contemporaine en Allemagne après 1989), voir note 6, op. cit. p. 59.

26 Hans-Ulrich Wehler, "Von der Herrschaft zum Habitus" (De la domination à l'habitus), Die Zeit, $n^{\circ} 44$ du 25.10.1996, p. 46. 
méthodologique sur la genèse et la structure de la conscience historique, en savent plus sur les mécanismes selon lesquels un individu perçoit et interprète l'histoire, que ceux qui souhaitent exclure des sciences historiques la problématique du monde de la vie, au motif qu'elle serait étrangère à la discipline historique, et dont la maxime scientifique est qu'on ne peut effectuer de travail de recherche historique qu'après avoir écarté tout élément subjectif ${ }^{27}$.

De véritables réformes de l'enseignement de l'histoire (qui dans ce cas s'appellerait peut-être autrement) et de la formation des enseignants pourraient naître de cette prise de position théorique et scientifique et de ses conséquences, à la fois au niveau structurel et organisationnel. La tendance immanente des sciences historiques à l'interdisciplinarité, en tant que discipline scientifique, permettrait de concevoir plus aisément un cursus d'étude dans lequel existerait une relation équilibrée entre le domaine spécifique et l'intégration aux autres disciplines; un cursus, donc, faisant aussi appel aux disciplines voisines et qui serait, lui-même, un pôle d'attraction pour les autres spécialités. Cette nouvelle matière pourrait avoir un effet bénéfique sur l'emploi du temps scolaire, généralement surchargé, avec ses matières canoniques, compartimentées, qu'elle allégerait. Des occasions beaucoup plus variées de découvrir quel sens potentiel peut avoir l'histoire du genre humain, de trouver les repères dont ils ont tellement besoin à l'heure actuelle, pour s'orienter, s'offriraient aux adolescents ${ }^{28}$.

Il serait présomptueux de tenter de faire un pronostic. Faut-il s'attendre à une phase de " restauration ", faisant barrage aux révolutions et aux réformes? Ou bien le besoin de réformes d'un grand nombre de personnes en Allemagne va-t-il parvenir à l'emporter ${ }^{29}$ ? La confédération de ceux qui partagent cette opinion n'est plus séparée, aujourd'hui, par l'ancienne frontière EstOuest. La frontière passe à travers l'Allemagne et s'étend bien au delà. Il est temps de retrouver le chemin de la sortie.

Peut-être l'Ange de l'Histoire, effrayé, peut-il céder la place à un autre symbole: Clio, telle qu'elle est représentée sur un tableau datant de 1592. Sur ses genoux, elle tient le Livre, à une certaine distance d'elle-même. Elle ne manifeste aucune inquiétude. Le calme revient. Ne considèrerait-t-elle pas l'Histoire avec une certaine ironie ? Ne serait-elle pas une idéaliste déçue?

27 Jörn Rüsen, Historische Orientierung. Über die Arbeit des Geschichtsbewußtseins, sich in der Zeit zurechtzufinden (Comment s'orienter en histoire. A propos du travail que la conscience de l'histoire opère pour trouver ses marques dans l'époque), Cologne, 1994.

28 Dagmar Klose, "Geschichtsunterricht in der Krise - ein gesamtdeutsches Problem " (L'enseignement de l'histoire en crise - un problème qui concerne toute l'Allemagne), Interdisziplinäres Zentrum für Lern- und Lehrforschung (Centre interdisciplinaire de recherche sur l'enseignement et les modes d'apprentissage), Université de Postdam, Comptes rendus LLF, n 11, nov. 1995, p. 108-120.

29 Ludger Kühnhardt, Revolutionszeiten. Das Umbruchjahr 1989 im geschichtlichen Zusammenhang (Une période révolutionnaire. En l'an 1989 : le bouleversement dans son contexte historique), Münich, 1994, p. 26. 